\title{
Clinical utility of echocardiography and magnetic resonance imaging for detecting cardiac complications in Systemic Sclerosis. A case report.
}

\author{
Bianca Olivia Cojan Minzat', Carmen Cionca ${ }^{2}$, Isabella Mihalcea ${ }^{1}$, Silvia Lupu ${ }^{3}$, \\ Lucia Agoston-Coldea ${ }^{1,2}$
}

${ }^{1} 2^{\text {nd }}$ Internal Medicine Department, "Iuliu Hatieganu" University of Medicine and Pharmacy, Cluj-Napoca, ${ }^{2}$ Hiperdia Diagnostic Imaging Center, Cluj-Napoca, ${ }^{3}$ Cardiovascular Disease and Transplant Institute, University of Medicine and Pharmacy of Targu Mures, Romania

\begin{abstract}
Systemic sclerosis (SSc) is a chronic autoimmune disease that, beside skin involvement, may affect peripheral vessels and several organs, such as the lungs, kidneys or heart. Cardiac impairment usually becomes symptomatic in the late stages of the disease and is associated with poor prognosis. We report the case of a 80-year-old woman presenting with symptoms of heart failure, subsequently diagnosed with limited SSc. Cardiac function was evaluated using a combined approach based on echocardiography and cardiac magnetic resonance imaging with late gadolinium enhancement. This case shows that the late diagnosis of SSc may have fatal consequences.

Keywords: systemic sclerosis, echocardiography, cardiac magnetic resonance imaging
\end{abstract}

\section{Introduction}

Systemic sclerosis (SSc) is a chronic systemic autoimmune disease characterized by vasculopathy, diffuse fibrosis and immune abnormalities. Fibroproliferative changes in the vascular walls may eventually lead to obliteration of the vascular lumen, resulting in ischemia [1]. Clinical manifestations of SSc are variable and depend on disease type and organ involvement. Cardiac impairment is usually diagnosed quite late, as it is clinically silent for a long time, which leads to poor prognosis and increased mortality rates [2]. The reported prevalence ranged between 50 and $81 \%$ in studies in which the diagnosis was made on autopsy $[3,4]$, while the clinical prevalence ranged between 10 and $35 \%[2,5,6]$. Cardiac involvement may be either primary or secondary to the impairment of other organs such as the lungs

Received 16.12.2014 Accepted 30.01.2015

Med Ultrason

2015, Vol. 17, No 2, 262-265

Corresponding author: Lucia Agoston-Coldea

$2^{\text {nd }}$ Internal Medicine Department

2-4 Clinicilor, 400006, Cluj-Napoca, Romania

Phone: +40264591942 ; Fax: +40264599817

Email: luciacoldea@yahoo.com and kidneys, and may include all layers of the heart [6]. Primary SSc is known to be associated with myocardial fibrosis $[1,3,4]$, which occurs long before symptoms' onset [7]. These findings suggest that myocardial fibrosis is frequent, even in asymptomatic patients, and should be identified early in order to improve disease prognosis. Myocardial fibrosis, conduction system abnormalities, pulmonary hypertension and ischemia due to coronary artery impairment are common findings resulting in arrhythmias, conduction disturbances, heart failure and sudden cardiac death [8,9]. Imaging techniques may be useful for identifying patients with SSc and subsequent cardiac impairment. However, echocardiography or perfusion scan cannot accurately identify myocardial fibrosis. By contrast, cardiac magnetic resonance imaging (MRI) with late gadolinium enhancement (LGE) is quite useful for identifying myocardial fibrosis [10-12], while also providing the advantage of very accurate cardiac structure and function quantification [13].

We present the case of a patient diagnosed with limited SSc after the onset of the cardiac symptoms using a combined approach based on echocardiography, cardiac MRI and immunological findings. 


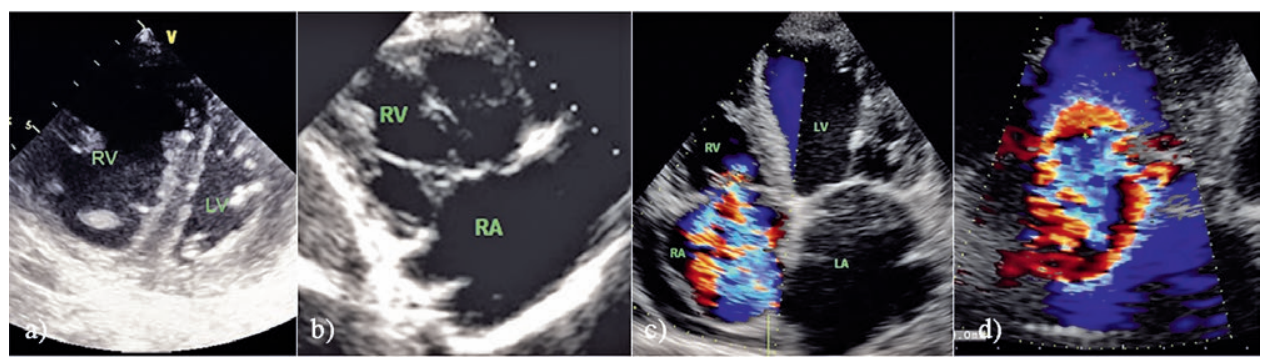

Fig 1. Transthoracic two-dimensional echocardiographic images: a) parasternal short-axis view showing an enlarged D-shaped right ventricle which suggests right ventricular volume and/or pressure overload due to pulmonary hypertension; b) parasternal long-axis view of right ventricle inflow showing dilated right chambers; $\mathrm{c}$ ) apical 4-chamber view showing severe tricuspid regurgitation and small pericardial effusion bordering the right atrium; d) quantitative assessment of tricuspid regurgitation - measurement of the proximal isovelocity surface area radius using the first aliasing limit. LA - left atrium, LV -left ventricle, RA - right atrium and RV - right ventricle.

\section{Case report}

A 80-year-old caucasian female was admitted to the $2^{\text {nd }}$ Internal Medicine Department due to rest dyspnea, orthopnea, constrictive chest pain, and swollen lower limbs that occurred two weeks prior to hospitalization and worsened progressively. The patient described Raynaud phenomena, during the last 15 years, but has not previously sought medical attention for that reason. Her medical history included high blood pressure and dyslipidemia (currently under treatment). Clinical examination revealed an altered general state, normal body temperature, overweight, telangiectasia, microstomia, sclerodactyly, indurate skin of the hand and forearms, edema of the lower limbs, and neck-vein distension. Auscultation revealed diminished murmur at the base of the right lung, regular cardiac rhythm (70 bpm), and ventricular gallop. Palpation revealed hepatomegaly. Spontaneous oxygen saturation, measured by plethysmography, was $82 \%$ and increased to $97 \%$ under oxygen therapy $\left(3 \mathrm{~L} / \mathrm{min} \mathrm{O}_{2}\right.$ ). An ECG was recorded at admission showing sinus rhythm, right axis deviation, $\mathrm{T}$ wave inversion in $\mathrm{V}_{1}-\mathrm{V}_{3}$, and an $\mathrm{S}_{1} \mathrm{Q}_{3} \mathrm{~T}_{3}$ pattern. Venous blood sample analysis showed elevated creatinkinase-MB and NT-proBNP levels, impaired renal function (creatinine clearance of $36 \mathrm{~mL} / \mathrm{min}$ ) and blood gas analysis indicated hypoxia. Echocardiography was performed, showing dilated right heart chambers, impaired right ventricle systolic function, severe tricuspid regurgitation, with increased systolic pulmonary artery pressure, and small pericardial effusion (fig 1); left ventricle ejection fraction was preserved and mitral flow analysis revealed impaired relaxation. Chest Xray showed small right pleural effusion, enlarged hilui, enlarged right chambers, dilated pulmonary artery, and aortic calcifications.
Based on the previous data, pulmonary embolism was suspected and an emergency thoracic spiral computer tomography scan was performed, showing a clot in the segmentary right pulmonary artery. Venous ultrasound of the inferior limbs was negative for deep vein thrombosis. As significant pulmonary embolism was excluded, based on clinical evidence, SSc was considered as an alternate cause for pulmonary hypertension and immunological tests were performed (Table I). According to ACR diagnosis criteria, limited SSc was established [14]. Cardiac MRI demonstrated right chamber enlargement, severely impaired right ventricle systolic function, linear LGE in the mid-wall region of the right ventricle free-wall and globular patchy LGE with indistinct borders at the lower insertion point of the left ventricular myocardium, suggesting myocardial fibrosis (fig 2). According to the preliminary severity scale [15] this case was regarded as severe, and therefore a poor prognosis with an increased risk of mortality was expected. The patient received anticoagulant treatment, diuretic and vasodilator drugs and was released having a significantly improved clinical status. Within the first month after discharge, the patient suffered cardiac arrest and could not be resuscitated.

Table I. Immunologic tests

\begin{tabular}{lll}
\hline Immunological markers & $\begin{array}{l}\text { Patient's } \\
\text { results }\end{array}$ & $\begin{array}{l}\text { Reference } \\
\text { values }\end{array}$ \\
\hline Anti-nuclear antibodies & $1: 1280$ & Negative \\
Anti-centromere antibodies & $839 \mathrm{U} / \mathrm{mL}$ & $<10 \mathrm{U} / \mathrm{mL}$ \\
Anticardiolipin antibodies & $1.5 \mathrm{U} / \mathrm{mL}$ & $<10 \mathrm{U} / \mathrm{mL}$ \\
Antifosfolipidic antibodies & $0.9 \mathrm{U} / \mathrm{mL}$ & $<10 \mathrm{U} / \mathrm{mL}$ \\
Anti SCL-70 antibodies & $2 \mathrm{U} / \mathrm{mL}$ & $<15 \mathrm{U} / \mathrm{mL}$ \\
Crioglobulins & Positive & Negative \\
Ig A & $4.41 \mathrm{~g} / \mathrm{L}$ & $0.7-4 \mathrm{~g} / \mathrm{L}$ \\
Ig G & $17.9 \mathrm{~g} / \mathrm{L}$ & $7-16 \mathrm{~g} / \mathrm{L}$ \\
Ig M & $2.36 \mathrm{~g} / \mathrm{L}$ & $0.4-2.3 \mathrm{~g} / \mathrm{L}$ \\
Rheumatoid factor & $64 \mathrm{U} / \mathrm{mL}$ & $<8 \mathrm{U} / \mathrm{mL}$ \\
\hline
\end{tabular}




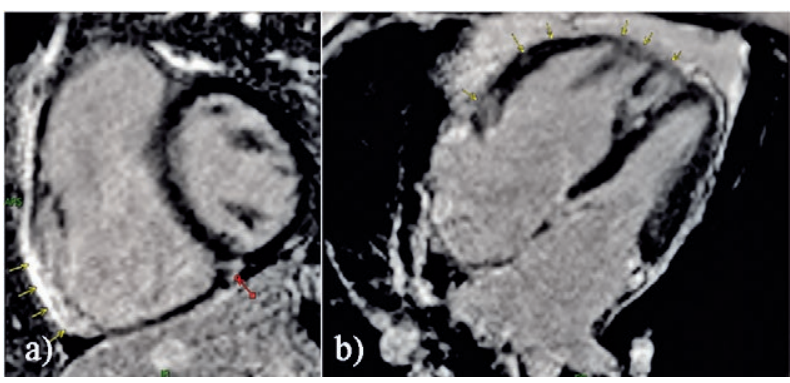

Fig 2. Cardiac late gadolinium enhancement magnetic resonance images: a) short-axis view and b) four-chamber horizontal long-axis view showing high signal predominating in the linear mid-wall late gadolinium enhancement (yellow arrows) region of the right ventricle free-wall and globular patchy enhancement with indistinct borders (red arrows) at the lower insertion point of the left ventricular myocardium, suggesting myocardial fibrosis. LGE - late gadolinium enhancement.

\section{Discussions}

In the currently presented case, the diagnosis of SSc was established after the onset of heart failure. The patient had a long history of uninvestigated Raynaud phenomenon. Clinical manifestations of SSc are extremely diverse and depend on disease type and organ involvement. Although subclinical cardiac involvement in SSc is common, clinical manifestations of cardiac involvement are fortunately rare [3]. Cardiac involvement in SSc may also lead to severe electric disturbances, such as arrhythmias or conduction abnormalities, pericardial effusion or constrictive pericarditis, increasing the risk for sudden cardiac death and affecting prognosis [2]. Heart failure may develop as a consequence of pulmonary hypertension and secondary cor pulmonale or due to diffuse fibrous replacement of cardiac muscles. Myocardial fibrosis is the hallmark of heart involvement in $\mathrm{SSc}$ and accounts for the majority of cardiac manifestations in these patients [10-12]. Although, echocardiography has been successfully used as a screening test for cardiac involvement in SSc patients, in some cases, cardiac changes remain undetected. By comparison, cardiac MRI is a more reliable and sensitive technique for diagnosing heart involvement in SSc by quantitative evaluation of the left and right ventricle and by analysing tissue characteristics, including inflammation and myocardial fibrosis $[10-12,16]$. Pulmonary artery hypertension is a frequent occurrence in patients with SSc, leading to poor prognosis and significantly increased mortality; it is characterized by abnormally elevated pulmonary blood pressure and increased pulmonary vascular resistance. Pulmonary artery hypertension occurs in about 7.85 to $12 \%$ of the patients with limited disease [17]. Survival in historical studies was exceedingly poor, with a 1-year survival of $45 \%$. Severe pulmonary artery hypertension was associated with a median survival of 12 months [18]. Limited disease has a relatively better prognosis except when pulmonary hypertension develops as a late complication. The early identification of patients with SSc-associated pulmonary arterial hypertension is very important, as it allows prompt initiation of therapy. In this particular patient, it is more than possible that primary thrombosis of the main pulmonary arteries has developed as a consequence of long standing pulmonary hypertension [19]. Early diagnosis and specific management of this severe complication, prior to the development of end-stage pulmonary vascular disease, are of utmost importance in SSc.

In conclusion, early and accurate detection of cardiac involvement should be a priority in SSc patients, as it is the most common reason for poor prognosis. Echocardiography is the first line imaging technique for the assessment of cardiac involvement, with good sensitivity and specificity, and can be completed by cardiac MRI to evaluate tissue characteristics. Cardiac MRI provides high spatial resolution, high reproducibility and low inter- and intraobserver variability, which render it superior to echocardiography in terms of ventricular size and function assessment and accurate diagnosis of myocardial fibrosis. These findings support the use of cardiac MRI as a screening method for cardiac impairment is SSc patients, as well as for risk stratification. However, echocardiography is non-invasive, easily performed and widely available. It can be routinely used, as it is relatively cheap and causes little discomfort to the patient. Doppler echocardiography in addition to clinical evaluation has been proposed for routine cardiac assessment, as it should detect all types of SSc cardiac complications.

\section{References}

1. Gilbane AJ, Denton CP, Holmes AM. Scleroderma pathogenesis: a pivotal role for fibroblasts as effector cells. Arthritis Res Ther 2013; 15: 215.

2. Ioannidis JP, Vlachoyiannopoulos PG, Haidich AB, et al. Mortality in systemic sclerosis: an international meta-analysis of individual patient data. Am J Med 2005; 118: 2-10.

3. D'Angelo WA, Fries JF, Masi AT, Shulman LE. Pathologic observations in systemic sclerosis (scleroderma). A study of fifty-eight autopsy cases and fifty-eight matched controls. Am J Med 1969; 46: 428-440.

4. Bulkley BH, Ridolfi RL, Salyer WR, Hutchins GM. Myocardial lesions of progressive systemic sclerosis. A cause of cardiac dysfunction. Circulation 1976; 53: 483-490.

5. Allanore Y, Meune C. Primary myocardial involvement in systemic sclerosis: evidence for a microvascular origin. Clin Exp Rheumatol 2010; 28: S48-S53. 
6. Ferri C, Valentini G, Cozzi F, et al. Systemic sclerosis: demographic, clinical, and serologic features and survival in 1,012 Italian patients. Medicine (Baltimore) 2002; 81: 139153.

7. Fernandes F, Ramires FJ, Arteaga E, Ianni BM, Bonfá ES, Mady C. Cardiac remodeling in patients with systemic sclerosis with no signs or symptoms of heart failure: an endomyocardial biopsy study. J Card Fail 2003; 9: 311-317.

8. Maione S, Cuomo G, Giunta A, et al. Echocardiographic alterations in systemic sclerosis: a longitudinal study. Semin Arthritis Rheum 2005; 34: 721-727.

9. Meune C, Avouac J, Wahbi K, et al. Cardiac involvement in systemic sclerosis assessed by tissue-doppler echocardiography during routine care: A controlled study of 100 consecutive patients. Arthritis Rheum 2008; 58: 1803-1809.

10. Bezante GP, Rollando D, Sessarego M, et al. Cardiac magnetic resonance imaging detects subclinical right ventricular impairment in systemic sclerosis. J Rheumatol 2007; 34: 2431-2437.

11. Tzelepis GE, Kelekis NL, Plastiras SC, et al. Pattern and distribution of myocardial fibrosis in systemic sclerosis: a delayed enhanced magnetic resonance imaging study. Arthritis Rheum 2007; 56: 3827-3836.

12. Hachulla AL, Launay D, Gaxotte V, et al. Cardiac magnetic resonance imaging in systemic sclerosis: a cross-sectional observational study of 52 patients. Ann Rheum Dis 2009; 68: 1878-1884.
13. Pennell DJ, Sechtem UP, Higgins CB, et al. Clinical indications for cardiovascular magnetic resonance (CMR): Consensus Panel report. Eur Heart J 2004; 25: 1940-1965.

14. Van den Hoogen F, Khanna D, Fransen J, et al. 2013 classification criteria for systemic sclerosis: an American College of Rheumatology/European League against Rheumatism collaborative initiative. Arthritis Rheum 2013; 65: 27372747.

15. Medsger TA JR, Silman AJ, Stehen VD, et al. A disease severity scale for systemic sclerosis: development and testing. J Rheumatol 1999; 26: 2159-2167.

16. Di Cesare E, Battisti S, Di Sibio A, et al. Early assessment of sub-clinical cardiac involvement in systemic sclerosis (SSc) using delayed enhancement cardiac magnetic resonance (CE-MRI). Eur J Radiol 2013; 82: e268-e273.

17. Hachulla E, Gressin V, Guillevin L, et al. Early detection of pulmonary arterial hypertension in systemic sclerosis: a French nationwide prospective multicenter study. Arthritis Rheum 2005; 52: 3792-3800.

18. Mukerjee D, St George D, Coleiro B, et al. Prevalence and outcome in systemic sclerosis associated pulmonary arterial hypertension: application of a registry approach. Ann Rheum Dis 2003; 62: 1088-1093.

19. Agarwal PP, Wolfsohn AL, Matzinger FR, Seely JM, Peterson RA, Dennie C. In situ central pulmonary artery thrombosis in primary pulmonary hypertension. Acta Radiol 2005; 46: 696-700. 